Luis Daniel Umezawa Makikado

José Luis Flordelís Lasierra

José Luis Pérez-Vela

Juan Carlos Montejo González

\section{Nutrition support during extracorporeal membrane oxygenation (ECMO) in adults}

Received: 24 September 2013

Accepted: 27 September 2013

Published online: 9 October 2013

(C) Springer-Verlag Berlin Heidelberg and ESICM 2013

Dear Editor

We read with interest the article by Ferrie et al. [1] on nutritional support in adult patients receiving extracorporeal membrane oxygenation (ECMO). The authors retrospectively audited 86 patients who received ECMO [venovenous (VV) or venoarterial (VA) mode] and fed using protocols that emphasize early enteral nutrition (EN). It is believed that the hemodynamic alterations which occur in these patients can lead to serious complications, such as mesenteric ischemia. The main finding of this elegant study is that early EN can be well tolerated in such patients (average $79.7 \%$ of target nutrition).

However, some obstacles to adequate nutrition were identified, such as the use of propofol for sedation, and 18 patients required parenteral support due to EN intolerance. Intolerance did not differ between ECMO modes.

We would like to discuss our recent findings on this subject as a complement to this study by Ferrie et al. [1]. We have recently reported a prospective case series of early EN in seven patients receiving VA ECMO for severe hemodynamic failure unresponsive to conventional therapies [2], as well as a prospective study involving 37 cardiac surgery patients with hemodynamic failure [3]. Although some differences are present in these two studies regarding the applied nutrition protocol and definition of nutritional goal, we came to the same main conclusion: early EN is possible and not associated with serious complications. However, we did note significant energy deficits, especially in patients with a long stay in the ICU (>14 days). Complications such as abdominal distention, diarrhea, and constipation were frequent ( $>25 \%$ of included patients). These complications barely received any mention in the paper published by Ferrie et al. [1]. We would therefore like to ask the authors about their experience with energy balances using EN alone, as well as a more detailed description of EN-related complications.

Despite current recommendations regarding nutritional support in patients with hemodynamic failure [4], we agree with the authors that EN is possible in ECMO patients, always under proper medical supervision.
Although controversial, we also consider that EN is also feasible in cardiac surgery patients with hemodynamic failure [5]. More studies are clearly warranted to confirm these findings.

Conflicts of interest None.

\section{References}

1. Ferrie S, Herkes R, Forrest P (2013) Nutrition support during ECMO in adults a retrospective audit. Intensive Care Med. doi:10.1007/s00134-013-3053-2

2. Umezawa Makikado LD, Flordelís Lasierra JL, Pérez-Vela JL et al (2013) Early enteral nutrition in adults receiving venoarterial extracorporeal membrane oxygenation: an observational case series. JPEN 37:281-284

3. Flordelís Lasierra JL, Pérez-Vela JL, Umezawa Makikado LD et al (2013) Early enteral nutrition in postoperative cardiac surgery patients with haemodynamic failure. JPEN (in press)

4. Kreymanna KG, Berger MM, Deutzc NE et al (2006) ESPEN guidelines on enteral nutrition: intensive care. Clin Nutr 25:210-223

5. Thibault R, Pichard C, Wernermann J et al (2011) Cardiogenic shock and nutrition: safe? Intensive Care Med $37: 35-45$

L. D. Umezawa Makikado (®) •

J. L. Flordelís Lasierra - J. L. Pérez-Vela •

J. C. Montejo González

Intensive Care Medicine Service, Hospital Universitario "12 de Octubre", Madrid, Spain

e-mail: luis_umezawa@hotmail.com 